\title{
Psychoedukation ist ein überholtes paternalistisches Konzept - Kontra
}

\author{
Psychoeducation is an Outdated Paternalistic Concept - Contra
}

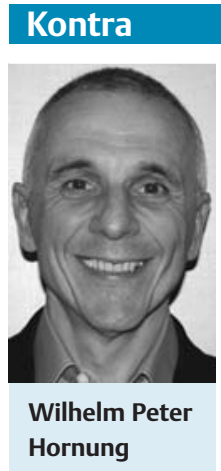

So mancher Leser mag sich wundern, wenn er diese Fragestellung zum Thema Psychoedukation liest. Psychoedukation paternalistisch? Psychoedukation gar anachronistisch?

Wieso ist man auf diese Frage gekommen? Was verbirgt sich dahinter? Hat man nahezu 30 Jahre nach den Arbeiten von Hogarty et al. [1] bereits vergessen, zu welchen Verbesserungen im Krankheitsverlauf schwer und chronisch psychisch Erkrankter psychoedukative Interventionen führen können? Schnell mag man argumentieren, dass psychoedukative Maßnahmen lediglich dazu beitrügen, das Wissen der Patienten in Bezug auf ihre Erkrankung zu verbessern. Den Kranken bringe man nur bei, die medizinischen Krankheitsmodelle zu übernehmen. Sie sollten gar dazu „erzogen“ werden, die für die Behandlung ihrer psychischen Störung erforderlichen Medikamente regelmäßig einzunehmen. Psychoedukativ tätige Therapeuten folgten damit den Interessen der pharmazeutischen Industrie.

Denn in der heutigen modernen Psychiatrie stehe das Empowerment der Patienten im Mittelpunkt psychiatrisch-psychotherapeutischen Handelns. Es gehe darum, „Recovery“ der schwer psychisch Kranken zu erzielen. Psychoedukation stehe diesen Ansätzen diametral gegenüber, wenn sie nicht solche Behandlungsziele sogar behindere.

Wer so argumentiert, hat das Wesen psychoedukativer Interventionen und die damit verbundene psychotherapeutische Haltung nicht verstanden.

Das von Anderson [2] eingeführte Kunstwort „Psychoedukation“ legt allerdings Begriffe wie Erziehung und Pädagogik nahe. Diese sind tatsächlich vielen Patienten sowie deren Angehörigen suspekt, zumal, wenn sie mit veralteten hierarchischen
Beschulungsmethoden in Verbindung gebracht werden. Solche Aversion ist berechtigt. Jedoch haben sich pädagogische Prinzipien verändert und im schulischen Bereich modernen Methoden Platz gemacht. Andererseits sind erzieherische Maßnahmen weder Inhalt noch Ziel psychoedukativen Handelns. Sie wären kontraproduktiv und sind vom psychoedukativen Psychotherapeuten auch nicht gewollt. Im Gegenteil soll durch Psychoedukation die Hierarchie der paternalistischen Therapieverordnung „von oben“ ersetzt werden durch ein partnerschaftliches Konzept, das basal („ganz unten“) ansetzt im Sinne einer Alphabetisierung der zunächst in Sachen „Psychose“ noch wenig informierten Erkrankten.

Hier muss konzidiert werden, dass nicht wenige als psychoedukativ bezeichnete Programme rein edukativ aufgebaut sind. Diese sind sehr schlicht gestaltet, umfassen oft nur wenige Sitzungseinheiten und beschränken sich tatsächlich im Wesentlichen auf die Vermittlung von krankheitsbezogenem Wissen (manchmal nur auf das Austeilen von schriftlichem Informationsmaterial). Sie stellen nicht den Regelfall dar und sollen daher nachfolgend unerwähnt bleiben.

\section{Psychoedukation ist patienten-} und angehörigenfokussiert

Psychoedukation im richtig verstandenen Sinne soll dazu führen, „Patienten und ihre Angehörigen über die Krankheit und ihre Behandlung zu informieren, das Krankheitsverständnis und den selbstverantwortlichen Umgang mit der Krankheit zu fördern und sie bei der Krankheitsbewältigung zu unterstützen“, wie im Konsensuspapier der Arbeitsgruppe Psychoedukation ausgeführt wird [3].

Der vielzitierte Dialog/Trialog auf „gleicher Augenhöhe“ gerät zur Farce, wenn den Betroffenen nicht die Chance gegeben wird, den natürlicherweise bestehenden Wissensvorsprung der professionellen Helfer bezüglich der psycho-physiologischen Hintergründe der Erkrankung und den sich daraus ergebenden Behandlungskonsequenzen auszugleichen. Das Expertentum der Betroffenen in Sachen subjektives Erfahrungswissen kann erst dann richtig zum Tragen kommen, wenn neben dem individuellen eigenen Krankheitserleben ein zusätzliches, übergeordnetes Wissen bezüglich allgemeingültiger Krankheitsfakten entsteht.

\section{Psychoedukation ist psychothera-} peutisch und ressourcenorientiert Bedeutsam bei diesem Vorgehen ist daher, dass mit einer psychotherapeutischen Haltung vorgegangen wird, welche die individuelle Position jedes Einzelnen erkennt, sie respektiert und die möglichst vollständige und langanhaltende Remission erreichen möchte. Dabei verbündet man sich mit dem Patienten, erarbeitet gemeinsame Ziele und versucht, diese im gemeinsamen Handeln zu erreichen. Es wäre falsch, unrealistische Heilsversprechungen zu geben, man sollte aber die restitutio ad integrum nie aus dem Auge verlieren. Diesem Ziel können sich Patienten und deren Angehörige auch dadurch selbstbestimmter nähern, dass sie im Rahmen der Psychoedukation über alle diesbezüglichen Möglichkeiten, aber auch die Begrenztheiten oder Risiken umfassend aufgeklärt werden [4]. So können Betroffene von einer informierten Warte aus bewusste und selbstbestimmte Entscheidungen in Bezug auf ihre Erkrankung (und ggf. deren Behandlung) treffen. Andernfalls wären solche Entscheidungen „Angst-getriggert“, weil aus Unwissenheit und auf Vorurteilen basierenden Ängsten heraus getroffen.

Psychoedukation legt somit das Fundament für ein weitestgehend gleichberechtigtes Miteinander von Therapeut und Patient (und, soweit möglich und gewünscht, den Angehörigen). Darin zeigt sich der trialogische Aspekt psychoedukativen Handelns [5].

Dass sich der Aspekt von gegenseitiger Achtung, Selbst- und Mitbestimmung der Patienten sich sogar bis in das konkrete 
Handeln im Rahmen der Psychopharmakotherapie hinein auswirkt, konnte empirisch aufgezeigt werden [6].

Psychoedukation ist evidenzbasiert Überhaupt liegen zahlreiche Befunde dafür vor, dass psychoedukative Interventionen auf vielfältigen Ebenen günstige Auswirkungen haben. Die Liste dieser zum Teil nachhaltigen Effekte kann der Kürze dieser Darstellung wegen nur quasi tabellarisch erfolgen: erwartungsgemäß wird bei Patienten und Angehörigen das Wissen über die zugrunde liegende Erkrankung erweitert. Verbessert werden bei den Patienten aber auch die Behandlungskontinuität („Compliance“), die Zufriedenheit mit der Therapie, der psychopathologische Befund, die soziale Adaptation, vordem ungünstige Krankheitskonzepte, aber auch Lebensqualität, Selbstkonzepte und subjektive Befindlichkeiten. Und diese günstigen Einflüsse von Psychoedukation machen bei den Patienten nicht halt. Sie finden sich nachweislich auch in Bezug auf die emotionale Familienatmosphäre sowie die psychosomatische Befindlichkeit, Lebensqualität und Empowerment bei den Angehörigen [7].

Ein besonders entscheidender Effekt ist derjenige auf die Rezidiv- und Rehospitalisierungsraten. Dass eine stationäre Wiederaufnahme nach Möglichkeit vermieden werden sollte, versteht sich im Sinne der Patienten von selbst. Psychoedukative Therapien (in Verbindung mit einer adäquaten Medikation) haben dies in vielen kontrollierten Studien unter Beweis gestellt. Laut neuesten Cochrane-Reviews ließ sich dies weltweit an über $1200 \mathrm{~Pa}$ tienten nachweisen [8]. Auch Langzeitstudien zeigten signifikante Effekte bifokaler
Psychoedukation sogar über einen 5- bis 8-Jahres-Zeitraum nach Therapieende $[9,10]$.

Psychoedukation ist folglich modern und zeitgemäß. Sie stellt einen bedeutsamen Zugang zum Patienten dar und nimmt ihn als gleichberechtigten Partner im Behandlungsgeschehen an. Sie bildet die Basis für alle weiteren Bemühungen im therapeutischen Prozess und fördert den Bewältigungsprozess psychischer Störungen, die früher noch als kaum behandelbar oder gar unheilbar galten.

\section{Literatur}

1 Hogarty GE, Anderson CM, Reiss DJ et al. Family psychoeducation, social skills training, and maintenance chemotherapy in the aftercare treatment of schizophrenia I. Oneyear effects of a controlled study on relapse and expressed emotion. Arch Gen Psychiatry 1986; 43: 633-642

2 Anderson CM, Hogarty GE, Reiss DJ. Family treatment of adult schizophrenic patients: a psychoeducational approach. Schizophr Bull 1980; 6: 490-515

3 Bäuml J, Pitschel-Walz G, Hrsg. Psychoedukation bei schizophrenen Erkrankungen. Konsensuspapier der Arbeitsgruppe „Psychoedukation bei schizophrenen Erkrankungen“. Stuttgart: Schattauer; 2008

4 Berger $H$. Die Förderung der Selbsthilfe theoretische Grundlagen und daraus abgeleitete Strategien zur Förderung und Wiederherstellung seelischer Gesundheit. In: Bäuml J, Pitschel-Walz G, Berger H, et al. Hrsg. Arbeitsbuch PsychoEdukation bei Schizophrenie (APES). Stuttgart: Schattauer; 2010: 166 - 171

5 Bender $M$. Individualisierung und trialogische Dimension. In: Bäuml J, Pitschel-Walz G, Hrsg. Psychoedukation bei schizophrenen Erkrankungen. Konsensuspapier der Arbeitsgruppe „Psychoedukation bei schizophrenen Erkrankungen. Stuttgart: Schattauer; 2008: 130-139
6 Hornung WP. Psychoedukation und Psychopharmakotherapie. Zur Kooperation schizophrener Patienten. Stuttgart: Schattauer; 1998

7 Pitschel-Walz G, Rummel-Kluge C, Froböse T et al. Steigerung des „empowerment“ bei Angehörigen von schizophren Erkrankten. Psychotherapeut 2012; 57: 313-318

8 Xia J, Merinder LB, Madhvi R. The Cochrane Collaboration. Winheim: John Wiley and Sons Ltd; 2011

9 Hornung WP, Feldmann R, Klingberg $S$ et al. Long-term effects of a psychoeducational psychotherapeutic intervention for schizophrenic outpatients and their key-persons - Results of a five-year follow-up. Eur Arch Psychiatry Clin Neurosci 1999; 249: $162-$ 167

10 Bäuml J, Pitschel-Walz G, Volz A et al. Psychoeducation in schizophrenia: 7-year followup concerning rehospitalization and days in hospital in the Munich Psychosis Information Project Study. J Clin Psychiatry 2007; 68: $854-861$

Sie haben eine eigene Meinung zu diesem Thema? Dann schreiben Sie uns an: psychiat-praxis@thieme.de!

Korrespondenzadresse

\section{Prof. Dr. med. Wilhelm Peter Hornung}

Arzt für Psychiatrie und Psychotherapie, Psychiatrie und Neurologie, Psychotherapeutische Medizin, Chefarzt Abt. Psychiatrie und Psychotherapie 1, LVR-Klink Bonn

Kaiser-Karl-Ring 20

53108 Bonn

wp.hornung@lvr.de

Bibliografie

DOI http://dx.doi.org/

10.1055/s-0034-1387485

Psychiat Prax 2015; 42: 297-298

(c) Georg Thieme Verlag KG

Stuttgart · New York

ISSN 0303-4259 